\title{
Prevalence of Congenital Malaria in Kisangani, A Stable Malaria Transmission Area in Democratic Republic of the Congo
}

\author{
Labama Otuli Noël $\mathbb{D D}^{1}{ }^{1}$ Bosenge Nguma Jean-Didier, ${ }^{1}$ Maindo Alongo Mike-Antoine, ${ }^{1}$ \\ Katenga Bosunga Gedeon, ${ }^{1}$ Mbo Mukonkole Jean-Paulin, ${ }^{2}$ Losimba Likwela Joris, ${ }^{3}$ \\ and Manga Okenge Jean-Pascal ${ }^{1}$
}

\author{
${ }^{1}$ Obstetrics and Gynecology Department, Faculty of Medicine and Pharmacy, University of Kisangani, \\ Kisangani, Democratic Republic of the Congo \\ ${ }^{2}$ Internal Medical Department, Faculty of Medicine and Pharmacy, University of Kisangani, \\ Kisangani, Democratic Republic of the Congo \\ ${ }^{3}$ Public Health Department, Faculty of Medicine and Pharmacy, University of Kisangani, \\ Kisangani, Democratic Republic of the Congo
}

Correspondence should be addressed to Labama Otuli Noël; nlabama@gmail.com

Received 25 September 2019; Revised 20 January 2020; Accepted 22 January 2020; Published 25 February 2020

Academic Editor: Faustino R. Perez-Lopez

Copyright ( 92020 Labama Otuli Noël et al. This is an open access article distributed under the Creative Commons Attribution License, which permits unrestricted use, distribution, and reproduction in any medium, provided the original work is properly cited.

\begin{abstract}
Background. Gestational malaria is a major public health problem. It produces fetal complications such as low birth weight, perinatal mortality, and congenital malaria. The present study is aimed at determining the prevalence of congenital malaria and its neonatal complications in the city of Kisangani. Methods. We conducted a cross-sectional study in Kisangani from 1 January to 30 September 2018. Our study population was composed of 1248 newborns born in our study sites, during the period of our study. Just after their birth, we performed the thick drop smear in the placental print and in umbilical blood smear. Results. The prevalence of congenital malaria was $13.98 \%$; $69.23 \%$ of newborns who contracted congenital malaria were from 18 - to 34 -yearold mothers, $53.85 \%$ from primiparous mothers, $92.31 \%$ from mothers who took intermittent preventive treatment in pregnancy with Sulfadoxine-Pyrimethamine, all (100\%) from mothers using the insecticide-treated mosquito nets and $7.69 \%$ from HIV-positive mothers. Low birth weight and perinatal mortality were recorded in $76.92 \%$ and $7.69 \%$ of congenital malaria cases, respectively. Intermittent preventive treatment in pregnancy with Sulfadoxine-Pyrimethamine had no effect on congenital malaria $(\mathrm{FE}=0.5218$; OR: $0.8,95 \% \mathrm{CI}: 0.1651-3.8769)$ and on low birth weight $(\mathrm{FE}=0.3675$; OR: $1.2308,95 \% \mathrm{CI}$ : $0.0037-$ 0.1464 ); however, it seemed to have protective effect against perinatal mortality ( $\mathrm{FE}=0.0001$; OR: $0.0233,95 \% \mathrm{CI}$ : 0.0037 0.1464). Conclusion. Congenital malaria remains a major problem in stable malaria transmission area like Kisangani, and it is grafted by major perinatal complications, particularly low birth weight and perinatal mortality. We recommend an extended study to clarify the relationship between the outcome of pregnancy and the intermittent preventive treatment in pregnancy with Sulfadoxine-Pyrimethamine.
\end{abstract}

\section{Background}

Malaria, known since antiquity, remains a public health problem. In the world, in 2010, the population likely to be infected by parasite and to develop disease was 3.2 billion, and the risk was high (more than one out of 1000 chance to contract malaria over a year) for 1.2 billion people [1]. After an unprecedented period of success in the world fight against malaria, the World Health Organization (WHO) reported that malaria-related mortality had remained the same from 2014 to 2016. In 2016, 216 million cases of malaria were reported in 91 countries, an increase of 5 million over the previous year. The number of related deaths has reached 445000, almost like in 2015 [2].

According to the WHO, the African region accounts for $90 \%$ of malaria cases and related deaths worldwide [2]. 
Children under five and pregnant women are the main victims $[3,4]$.

Every year in Africa, more than 30 million pregnant women live in malaria-endemic areas. The prevalence of malaria during pregnancy is variable in endemic area. It varies from 5 to $40 \%$ depending on the country [5]. Malaria infestation of pregnant women is a major public health problem, as this disease is a threat to themselves and their children, with up to 200000 stillborn babies each year due to the presence of malaria during pregnancy. In addition to perinatal mortality, it also causes low birth weight and congenital malaria [2].

The Democratic Republic of Congo (DRC) is the second sub-Saharan African country most affected by malaria after Nigeria. These two countries alone, added to India, account for $40 \%$ of malaria cases [6]. In northeast DRC where Tshopo province is located, malaria is the cause of consultation in $37 \%$ of all pathologies and the cause of death at $30 \%$ of cases $[4,5]$. The equatorial facies which dominates this province in general and the city of Kisangani in particular and the presence of Plasmodium falciparum which ensures intense and permanent transmission as well as the insanitary condition of the environment would be the cause.

According to the DRC National Malaria Control Program (NMCP), some factors, including the late and irregular attendance of prenatal consultations (ANC) by pregnant women and the shortage of stock of SulfadoxinePyrimethamine in healthcare settings, make it difficult to control malaria among pregnant women in the DRC [7].

These factors make the prevalence of gestational malaria high in Kisangani, a stable malaria transmission area. In areas of stable transmission of malaria, ovular complications predominate [8]. Therefore, the risk of congenital malaria would not be excluded.

In conducting this study, we set ourselves the goal of raising the prevalence of congenital malaria and its complications in Kisangani City.

\section{Methods}

2.1. Study Sites. This study was conducted in the General Reference Hospitals of Kabondo, Lubunga, and MakisoKisangani and in the Reference Healthcare Centers Foyer, Matete, and Saint Joseph: all located in the city of Kisangani, a malaria-endemic area in northeastern DRC. These firstlevel medical structures were selected on the basis of their high attendance and the representativeness of the communes in the city of Kisangani. However, Kisangani commune was not represented because it does not have any first-level hospitals. Thus, we selected General Reference Hospital Kabondo, a second medical training in the commune of Makiso which, for reasons of proximity, receives most of the women residing in Kisangani commune.

2.2. Type and Study Period. It is a cross-sectional study from 1 January to 30 September 2018.

2.3. Sample Size and Study Population. The study population was composed of all newborns who were born in our study sites, during the period of our study.
Newborns from parturients living in and around Kisangani (less than $30 \mathrm{~km}$ ) at least 14 days before delivery and without history of taking antimalarial drugs during the same period were included in the study.

To calculate the minimum size of our sample, we used the STATCALC software Epi info 7.2.2.6. Based on the 14.8\% of placental malaria cases found in a pilot study conducted by Lukuka et al. [9] in 4 maternity wards in the city of Kinshasa (DRC) which has the same epidemiological aspect as the city of Kisangani, with 95\% confidence level and an acceptable $5 \%$ margin of error, we found a minimum sample size of 194 cases. For our study, 1248 parturients, who complied with the inclusion criteria, were recruited consecutively. There were no twins among their newborns.

2.4. Data Collection. Data collection was prospective. It was done when the mothers of our babies surveyed came to give birth. To collect the data for this study, we used a previously established data collection form. At the admission of parturients to the labor room, the investigators (nurses or midwives) proceeded to interview the parturients. During the interview, the investigators obtained their informed consent for the participation in the study, the attribution of a unique code to each parturient and investigations, related to their socioanthropometric parameters (age, education level, occupation, and common residence), their background and preventive measures against malaria, in particular, using of the insecticide-treated mosquito nets (ITNs) and intermittent preventive treatment in pregnancy with SulfadoxinePyrimethamine (IPTp-sp). The code assigned to each parturient included the initial of her healthcare structure and the initial of her arrival order at the delivery facility. The same parturient code was attributed after delivery to her newborn, but followed by the suffix NN.

The interview was followed by the full physical examination of these parturients. At the same time, to assess the nutritional status of parturients, their mid-upper arm circumference (MUAC) was measured. Thus, in accordance with the technical files of National Nutrition program of the DRC [10], a MUAC $\geq 22 \mathrm{~cm}$ was considered as a normal nutritional status, a MUAC entry $\geq 21$ to $<22 \mathrm{~cm}$ as mild malnutrition and a MUAC $<21 \mathrm{~cm}$ was considered as severe malnutrition.

Immediately after delivery, nurses or midwives thoroughly examined completely newborns while weighing them using the $\mathrm{SH}-8008$ electronic baby scale. Referring to the WHO definition of low birth weight [11], a birth weight lower than $2500 \mathrm{gr}$ at birth was considered as low birth weight (LBW). The laboratory technicians of the study performed the thick drop smear (TDS) in placental prints and umbilical cord blood.

\subsection{Laboratory Investigations}

2.5.1. Training. The laboratory technicians had been briefed on techniques of collecting peripheral blood, blood from placental prints, and umbilical cord blood from the newborn and the techniques of spreading, preparing, and conveying samples according to the guidelines of the NMCP. They have 
also been trained on testing HIV/AIDS and prevention of other sexually transmitted diseases as recommended by the National AIDS Control Program (NACP).

2.5.2. HIV Test. Upon admission of the parturients to the labor room, after obtaining their informed consent, the laboratory technicians collected $2 \mathrm{ml}$ of venous blood in an EDTA tube. They then performed rapid HIV test with the rapid test Determine assay HIV. Positive cases were referred to the provincial laboratory of the NACP in Kisangani for confirmation of the diagnosis, whereby all HIV-positive mothers were subjected with their newborns to antiretroviral treatment according to the DRC policy related to HIV prevention and care [12].

2.5.3. Malaria Diagnosis. Immediately after delivery, after clamping and cleaning the umbilical cord, laboratory technicians took $1 \mathrm{ml}$ of blood by puncture of the umbilical vein and immediately spread it on a slide. Then, to perform the placental print, they cleaned the placenta superficially, incised its maternal surface, and applied a blade on the incised placental surface. These slides, umbilical blood smears and placental print, were stained for 10 minutes with $10 \%$ diluted Giemsa solution, before being sent to the Provincial Public Health Laboratory (PPHL) for microscope reading at the objective $100 \mathrm{x}$. The diagnosis of malaria parasitaemia was considered when trophozoites or other plasmodium development stages were present in the sample. Thus, placental malaria was defined as the presence of malaria parasitaemia in the placental print, whereas congenital malaria was defined as the presence of malaria parasitaemia in umbilical blood smear among newborns who were born from mothers with placental malaria.

For the quality control of the results obtained at the PPHL, $10 \%$ of random positive samples and $10 \%$ of random negative samples were transferred and analyzed at the National Institute of Biomedical Research in Kinshasa; and the concordance rate, by calculating the Kappa index, was $85 \%$ for the same analyses.

2.6. Statistical Analysis. The collected data was encoded in Excel and imported for analysis using STATCALC software Epi info 7.2.2.6. We described the sample by calculating the mean, the frequency, and the percentage, as well as the averages and their standard deviations.

To compare proportions, we calculated Pearson's chi squared or fisher's exact test at the level of significance $<0.05$.

To measure the strength of the association, the odds ratio (OR) and its 95\% confidence interval (CI) were determined.

2.7. Ethical Considerations. Before conducting this study, we submitted the protocol to the ethics committee of Kisangani University and obtained its approval (Approval No. UNIKIS/CER/006/2018). To participate in the study, mothers of newborns had to sign the informed consent form. All newborns and mothers in whom the TDS was positive were subjected to antimalarial treatment according to the PNLPrecommended regimen in the DRC.

\section{Results}

3.1. General Characteristics of Newborns'Mothers $(N=1248)$. The mean age of newborns' mothers was $25.40 \pm 6.24$ years; 170 newborns' mothers $(13.62 \%)$ were under the age of 19 ; $24.36 \%$ were primiparous; $95.03 \%$ of them used the ITNs; 95.27\% used IPTp-sp; the proportion of respondents with positive HIV serology was $3.37 \%$; 186 (14.90\%) had placental malaria.

3.2. Characteristics of Newborns' Mothers Who Had Placental Parasitaemia. We notice that 128 newborns' mothers who had placental malaria $(68.82 \%)$ were aged 19 to 34 while 52 $(27.96 \%)$ were under $19 ; 52.69 \%$ of them were primiparous; $174(93.55 \%)$ followed at least one ANC session and took at least one IPTp-sp dose; 168 (90.323\%) were using ITNs; $5.38 \%$ were HIV positive; the mean of their MUAC was $22.1812 \pm 1.4147$; and $90.86 \%$ of them had a normal nutritional status (MUAC $\geq 22 \mathrm{~cm}$ ) (Table 1 ).

3.3. Prevalence of Congenital Malaria. In 186 TDS performed in umbilical cord blood samples of newborns from mothers with placental malaria, 26 were positive, as a prevalence of congenital malaria of $13.98 \%$.

3.4. Profile of Mothers of Newborns Who Have Contracted Congenital Malaria. In Table 2, we described the profile of mothers of newborns who have contracted congenital malaria.

We found that $69.23 \%$ of newborns who have contracted congenital malaria were born from mothers aged 18 to 34 , $53.85 \%$ from primiparous mothers, $92.31 \%$ from mothers who took IPTp-sp; all newborns who have contracted congenital malaria (100\%) were born from mothers using ITNs and $7.69 \%$ from HIV-positive mothers (Table 2).

3.5. Perinatal Complications of Congenital Malaria. In Table 3, we described the complications recorded in the newborns who have contracted congenital malaria.

We found that $76 \%$ of newborns who have contracted congenital malaria had low birth weight. We recorded perinatal mortality in $7.69 \%$ of cases (Table 3 ).

3.6. Relationship between Taking IPTp-sp and the Outcome of Pregnancy. In Table 4, we determined the relationship between IPTp-sp and the outcome of pregnancy among parturients who had placental malaria.

While reading this table, we notice that, among parturients with placental malaria, taking IPTp-sp had no effect on congenital malaria ( $\mathrm{FE}=0.5218$; OR: 0.8, 95\% CI: 0.16513.8769) and LBW (FE $=0.3675$; OR: 1.2308 , 95\% CI: 0.0037-0.1464); however, it seemed to have protective effect against perinatal mortality $(\mathrm{FE}=0.0001$; OR: $0.0233,95 \%$ CI: 0.0037-0.1464) (Table 4).

\section{Discussion}

4.1. Prevalence of Congenital Malaria. In this study, the prevalence of congenital malaria in Kisangani was $13.98 \%$ $(95 \% \mathrm{CI}=9.34-19.81)$. The prevalence of congenital malaria varies from region to region or from a study to another, 
TABLE 1: Characteristics of newborns' mothers who had placental parasitaemia $(N=186)$.

\begin{tabular}{|c|c|c|}
\hline Newborns' mothers & $n(\%)$ & $95 \% \mathrm{CI}$ \\
\hline \multicolumn{3}{|l|}{ Age (years) } \\
\hline$<19$ & $52(27.96)$ & 21.64-34.99 \\
\hline $19-34$ & $128(68.82)$ & $61.63-75.39$ \\
\hline$\geq 35$ & $6(3.23)$ & $1.19-6.89$ \\
\hline \multicolumn{3}{|l|}{ Level of education } \\
\hline Illiterate & $8(7.20)$ & $1.87-8.30$ \\
\hline Primary & $32(4.30)$ & $10.32-26.89$ \\
\hline Secondary & $118(63.44)$ & $56.08-70.37$ \\
\hline Higher and university & $28(15.05)$ & $10.24-21.02$ \\
\hline \multicolumn{3}{|l|}{ Profession } \\
\hline Student & $16(8.89)$ & $5.17-14.03$ \\
\hline Housewife & $154(85.56)$ & $79.56-90.34$ \\
\hline Employee & $6(3.33)$ & $1.23-7.11$ \\
\hline Informal sector & $4(2.22)$ & $0.61-5.59$ \\
\hline \multicolumn{3}{|l|}{ Residence } \\
\hline Rural & $32(17.20)$ & $12.08-23.41$ \\
\hline Urban & $154(82.80)$ & $76.59-87.92$ \\
\hline \multicolumn{3}{|l|}{ Parity } \\
\hline 1 & $98(52.69)$ & $45.25-60.04$ \\
\hline 2 and more & $88(47.31)$ & $39.96-54.75$ \\
\hline \multicolumn{3}{|l|}{ ANC visits } \\
\hline 0 & $12(6.45)$ & $3.38-11.00$ \\
\hline 1 & $28(15.05)$ & $10.24-21.02$ \\
\hline 2 & $18(9.68)$ & $5.84-14.86$ \\
\hline 3 & $76(40.86)$ & $33.72-48.29$ \\
\hline 4 & $38(20.43)$ & $14.88-26.95$ \\
\hline 5 & $14(7.53)$ & $4.18-12.31$ \\
\hline \multicolumn{3}{|l|}{ IPTp-sp doses } \\
\hline 0 & $12(6.45)$ & $3.38-11.00$ \\
\hline 1 & $22(11.83)$ & $7.56-17.36$ \\
\hline 2 & $23(12.37)$ & $8.00-17.97$ \\
\hline 3 & $129(69.35)$ & $62.19-75.89$ \\
\hline \multicolumn{3}{|l|}{ Use of ITNs } \\
\hline No & $18(9.68)$ & $5.84-14.86$ \\
\hline Yes & $168(90.32)$ & $85.14-94.16$ \\
\hline \multicolumn{3}{|l|}{ HIV serology } \\
\hline Negative & $176(94.62)$ & $90.34-97.36$ \\
\hline Positive & $10(5.38)$ & $2.61-9.66$ \\
\hline \multicolumn{3}{|c|}{ MUAC $(\mathrm{cm})($ mean: $22.1812 \pm 1.4147)$} \\
\hline$\geq 22$ & $169(90.86 \%)$ & $85.77-94.59$ \\
\hline$\geq 21$ to $<22$ & $16(8.60 \%)$ & $5.00-13.59$ \\
\hline$<21$ & $1(0.54 \%)$ & $0.01-2.96$ \\
\hline
\end{tabular}

and it is often influenced by the methodology used to define the case. In this study, the population consisted of newborns of mothers with placental parasitaemia and congenital malaria was defined by positivity of the umbilical cord blood of these newborns.
TABle 2: Profile of mothers of newborns who have contracted congenital malaria $(N=26)$.

\begin{tabular}{|c|c|c|}
\hline Mothers & $n(\%)$ & $95 \% \mathrm{CI}$ \\
\hline \multicolumn{3}{|l|}{ Age (years) } \\
\hline$<19$ & $8(30.77)$ & $14.33-51.79$ \\
\hline $19-34$ & $18(69.23)$ & $48.21-85.67$ \\
\hline$\geq 35$ & $0(0.00)$ & - \\
\hline \multicolumn{3}{|l|}{ Parity } \\
\hline 1 & $14(53.85)$ & $33.37-73.41$ \\
\hline 2 and more & $12(46.15)$ & $26.59-66.63$ \\
\hline \multicolumn{3}{|l|}{ Taking IPTp-sp } \\
\hline No & $2(7.69)$ & $0.95-25.13$ \\
\hline Yes & $24(92.31)$ & $74.87-99.05$ \\
\hline \multicolumn{3}{|l|}{ Maternal fever } \\
\hline No & $24(92.31)$ & $74.87-99.05$ \\
\hline Yes & $2(7.69)$ & $0.95-25.13$ \\
\hline \multicolumn{3}{|l|}{ Using of ITNs } \\
\hline No & $0(0.00)$ & - \\
\hline Yes & $26(100)$ & $86.77-100$ \\
\hline \multicolumn{3}{|l|}{ HIV serology } \\
\hline Negative & $24(92.31)$ & $74.87-99.05$ \\
\hline Positive & $2(7.69)$ & $0.95-25.13$ \\
\hline
\end{tabular}

Table 3: Perinatal complications of congenital malaria $(N=26)$.

\begin{tabular}{lcc}
\hline Perinatal complications & $n(\%)$ & $95 \%$ CI \\
\hline Low birth weight & & \\
$\quad$ No & $6(23.08)$ & $8.97-43.65$ \\
Yes & $20(76.92)$ & $56.35-91.03$ \\
Perinatal mortality & & \\
No & $24(92.31)$ & $74.87-99.05$ \\
Yes & $2(7.69)$ & $0.95-25.13$ \\
\hline
\end{tabular}

The prevalence of congenital malaria that we found in Kisangani is higher than that found by Piñeros-Jiménez et al. [13] in Uraba in Colombia (2.5\%), by EnweronuLaryea et al. [14] among Ghanaian newborns (2.2\%), by Mosha et al. [15] in Morogoro in Tanzania (0.5\%; 95\% CI: $0.0-1.5 \%)$, by Mwaniki et al. [16] in Kenya (0.35\%), that reported by Fischer's investigation in Africa [17] (7\%), by Falade et al. [18] in Ibadan (2.6\%), in Ilorin (3.4\%), and in Kaduna $(8.5 \%)$. In contrast to us, these different authors had identified newborns without taking into account placental malaria parasitaemia. In addition to this reason, for Mwaniki et al. [16], congenital malaria was defined as the detection in newborns of asexual intraerythrocytic forms of plasmodium species in the first 7 days after birth.

Our result is lower than that of Bah et al. [19] who found a prevalence of congenital malaria of $15.57 \%$ in Guinea. In addition to the TDS in cord blood and placenta, like in ours, these authors also took into account the TDS in the heel to define the case of congenital malaria. 
TABLE 4: Relationship between taking IPTp-sp and the outcome of pregnancy $(N=186)$.

\begin{tabular}{|c|c|c|c|c|}
\hline \multirow{2}{*}{ Taking IPTp-sp } & \multicolumn{2}{|c|}{ Congenital malaria } & \multirow{2}{*}{$p$ value } & \multirow{2}{*}{ OR $(95 \% \mathrm{CI})$} \\
\hline & Yes & No & & \\
\hline No & $2(16.67 \%)$ & $10(83.33 \%)$ & $0.5218^{*}$ & $1.25(0.2579-6.0576)$ \\
\hline \multirow[t]{3}{*}{ Yes } & $24(13.79 \%)$ & $150(86.21 \%)$ & & 1 \\
\hline & \multicolumn{2}{|c|}{ Perinatal mortality } & & \\
\hline & Yes & No & & \\
\hline No & $4(3.23 \%)$ & $8(86.77 \%)$ & $0.0001^{*}$ & $0.0233(0.0037-0.1464)$ \\
\hline \multirow[t]{3}{*}{ Yes } & $2(2.19 \%)$ & $172(97.81 \%)$ & & 1 \\
\hline & & & & \\
\hline & Yes & No & & \\
\hline No & $6(50.00 \%)$ & $6(50.00 \%)$ & $0.3675^{*}$ & $1.2308(0.0037-0.1464)$ \\
\hline Yes & $96(55.17 \%)$ & $78(44.83 \%)$ & & 1 \\
\hline
\end{tabular}

The prevalence of our study is also less than the one found by Lehner and Andrews [20] in Guinea (14.6\%) and Obiajunwa et al. [21] in Nigeria (54.2\%). In Benin, Sagbo et al. [22] found $57 \%$ of congenital malaria in newborns and $17.08 \%$ in those whose mothers had malaria during pregnancy. To diagnose congenital malaria, Sagbo et al. [22] had considered the TDS and the malaria rapid test on the umbilical cord or peripheral blood of the newborn. Obiajunwa et al. [21] found a strong association between placental malaria and cord parasitaemia. Indeed, the prevalence of placental malaria parasitaemia was very high in their study. The high prevalence of congenital malaria recorded by us, as by these authors, would also be related to the fact that Kisangani is a stable malaria transmission zone.

4.2. Profile of Mothers of Newborns Who Have Contracted Congenital Malaria. We found that $69.23 \%$ of newborns who have contracted congenital malaria were born from mothers aged 18 to $34,53.85 \%$ from primiparous mothers, 92.31\% from mothers who took IPTp-sp; all newborns (100\%) were born from mothers using ITNs and 7.69\% from HIV-positive mothers.

As for age, our result is similar to that found by Noudamadjo et al. [23] who found that the age of newborns' mothers with congenital malaria was less than 30 years in $78.9 \%$ of cases and between 20 and 30 years in $66.7 \%$ of cases. Bah et al. [19] found an average age of newborns' mothers with congenital malaria of $26 \pm 14$ years with prevalence in the age group of 20-35 years (4.7\%) while Agossou et al. [24] had found an average age of $27.3 \pm 6$ years with extremes of 15 to 42 years. Vanga-Bosson et al. [25] found a median of 17 years.

Compared to parity, in our research, $53.85 \%$ of newborns with congenital malaria were born from primiparous mothers. This prevalence is lower than that reported by Agossou et al. [24] (62.9\%) and the one found by VangaBosson et al. [25] in Ivory Coast (100\%).

In our research, $92.31 \%$ of newborns with congenital malaria were born from mothers who completed ANC. This result does not agree with Bah et al. [19] who had diagnosed congenital malaria in $7.3 \%$ of newborns of mothers with at least $4 \mathrm{CPN}$.

We found that $92.31 \%$ of newborns who had had congenital malaria were born from mothers who took IPTp-sp. Fischer [17] found a prevalence of congenital malaria of $6.1 \%$ in newborns of mothers who took IPTp-sp. VangaBosson et al. [25] found a prevalence of 4.7\% (95\% CI: 1.3$11.6 \%)$.

In our research, $7.69 \%$ of our respondents were born to $\mathrm{HIV}$-positive mothers. In their study, Vanga-Bosson et al. [25] found that no newborn mother with congenital malaria was infected with HIV.

In a study conducted on the prevalence of gestational malaria in Kisangani, Labama et al. [26] found that the prevalence of gestational malaria was very high in Kisangani and was associated with younger age, primiparity and HIV and that monitoring ANC and taking IPTp-sp did not protect Kisangani's pregnant women from malaria. We believe that the findings made in that study justify our results.

4.3. Perinatal Complications of Congenital Malaria. In our study, $76 \%$ of newborns with congenital malaria had low birth weight. We recorded perinatal mortality in $7.69 \%$ of cases.

In a study in Ghanaian newborns, Enweronu-Laryea et al. [14] found that $55 \%$ of newborns with congenital malaria had a low birth weight.

These complications would be due to uteroplacental hypoperfusion. In malaria-endemic areas such as Kisangani, in case of malaria infection, plasmodiums are sequestered in the placenta [8]. This would be the cause of the decrease in uteroplacental perfusion, which would lead to intrauterine growth retardation, causing low birth weight. Decreased uteroplacental perfusion would also lead to fetal distress that may lead to perinatal mortality.

4.4. Relationship between Taking IPTp-sp and the Outcome of Pregnancy. Taking IPTp-sp had no effect on congenital malaria (FE =0.5218; OR: 0.8, 95\% CI: 0.1651-3.8769) and LBW ( $\mathrm{FE}=0.3675$; OR: 1.2308, 95\% CI: 0.0037-0.1464); 
however, it seemed to have protective effect against perinatal mortality ( $\mathrm{FE}=0.0001$; OR: 0.0233, 95\% CI: 0.0037-0.1464).

Taking IPTp-sp had no effect on congenital malaria and low birth weight because of the resistance of plasmodium to Sulfadoxine-Pyrimethamine, reported in DRC [27]. It was reported in a study conducted in Kisangani that IPTp-sp did not have an effect on the birth weight [28].

Because of the size of our sample with a small proportion of perinatal mortality, it is difficult to comment on the apparent protective effect of IPTp-sp against perinatal mortality. Indeed, the noneffect on congenital malaria and low birth weight, in a context of plasmodium resistance to Sulfadoxine-Pyrimethamine, would cause IPTp-sp to have no protective effect on perinatal mortality. This calls for a more extended study to clarify these facts.

\section{Conclusion}

Congenital malaria remains an important problem in areas of stable malaria transmission such as Kisangani. Its prevalence is $13.98 \%$ and it is grafted with major perinatal complications, particularly the low birth weight of newborns and perinatal mortality. We recommend an extended study to clarify the relationship between the outcome of pregnancy and the intermittent preventive treatment in pregnancy with Sulfadoxine-Pyrimethamine.

$\begin{array}{ll}\text { Abbreviations } \\ \text { ANC: } & \text { Antenatal care } \\ \text { CI: } & \text { Confidence interval } \\ \text { DRC: } & \text { Democratic Republic of Congo } \\ \text { FE: } & \text { Fisher exact } \\ \text { IPTp-sp: } & \text { Intermittent preventive treatment in pregnancy } \\ & \text { with Sulfadoxine-Pyrimethamine } \\ \text { ITNs: } & \text { Insecticide-treated mosquito nets } \\ \text { LBW: } & \text { Low birth weight } \\ \text { MUAC: } & \text { Mid-upper arm circumference } \\ \text { NACP: } & \text { National AIDS Control Program } \\ \text { NMCP: } & \text { National Malaria Control Program } \\ \text { OR: } & \text { Odds ratio } \\ \text { PPHL: } & \text { Provincial Public Health Laboratory } \\ \text { TDS: } & \text { Thick drop smear } \\ \text { WHO: } & \text { World Health Organization. }\end{array}$

\section{Data Availability}

All data used to support the findings of this study are available from the corresponding author upon request.

\section{Conflicts of Interest}

The authors declare that there is no conflict of interest.

\section{Authors' Contributions}

Dr. Labama conceived the research protocol and wrote the manuscript. Drs. Labama and Bosenge were responsible for data collection. Drs. Bosenge and Maindo encoded and processed the data and contributed to the literature review.
Professors Katenga, Mbo, and Losimba corrected the research protocol and enriched the manuscript. Professor Manga corrected the research protocol, enriched the manuscript, and validated the research. All authors validated the final version.

\section{Acknowledgments}

We thank the University of Kisangani, which is sponsoring this study. We would also like to thank the officers of hospitals which serve as our research site and the Provincial Public Health Laboratory of Kisangani where the parasitological analyses were carried out. We thank all pregnant women who agreed to participate in this research. Finally, we thank all the research team.

\section{References}

[1] World Health Organization, World Malaria Report 2011, WHO, Genève, 2011.

[2] World Health Organization, World Malaria Report 2017, WHO, Genève, 2017.

[3] World Health Organization, World Malaria Report 2018, WHO, Genève, 2018.

[4] S. Gabrielli, L. Bellina, G. L. Milardi et al., "Malaria in children of Tshimbulu (Western Kasai, Democratic Republic of the Congo): epidemiological data and accuracy of diagnostic assays applied in a limited resource setting," Malaria Journal, vol. 15, no. 81, 2016.

[5] "Programme national de lutte contre le paludisme," in Faire reculer le paludisme, Plan stratégique 2009-2011, PNLP, Ministère de la santé, RDC, 2009, http://www .nationalplanningcycles.org/sites/default/files/planning_ cycle_repository/democratic_republic_of_congo/malaria_ plan_strategique_2007_2011.pdf.

[6] World Health Organization, World Malaria Report 2008, WHO, Genève, 2008.

[7] Ministère du Plan et Suivi de la Mise en œuvre de la Révolution de la Modernité and Ministère de la Santé Publique, Enquête Démographique et de Santé en République Démocratique du Congo 2013-2014, MPSMRM, MSP et ISF International, Rockville, Maryland, USA, 2014, https://www.unicef.org/drcongo/ media/1046/file/EDS-RDC\%20II\%202013-2014.pdf.

[8] B. J. Okoko, G. Enwere, and M. O. C. Ota, "The epidemiology and consequences of maternal malaria: a review of immunological basis," Acta Tropica, vol. 87, no. 2, pp. 193-205, 2003.

[9] K. A. Lukuka, O. S. Fumie, M. R. Mulumbu, B. J. Lokombe, and T. J. J. Muyembe, "Prévalence du paludisme à l'accouchement dans quatre maternités de la ville de Kinshasa, République Démocratique du Congo," Bulletin de la Société de Pathologie Exotique, vol. 99, no. 2, pp. xxx-xxx, 2006.

[10] Programme National de Nutrition, Fiches techniques sur l'approche Nutrition Assessment Counseling and Support, PRONANUT, Ministère de la santé, RDC, 2016, https:// www.fantaproject.org/sites/default/files/resources/DRCNACS-Fiches-Tech-2016.pdf.

[11] World Health Organization, International statistical classification of diseases and related health problems, 10th edition, , 2010https://www.who.int. 
[12] Programme National de Lutte contre le SIDA et les IST, Guide de prise en charge intégrée du VIH en République Démocratique $d u$ Congo, PNLS, Ministère de la santé, RDC, 2016.

[13] J. G. Piñeros-Jiménez, G. Álvarez, A. Tobón, M. Arboleda, S. Carrero, and S. Blair, "Congenital malaria in Urabá, Colombia,” Malaria Journal, vol. 10, p. 239, 2011.

[14] C. C. Enweronu-Laryea, G. O. Adjei, B. Mensah, N. Duah, and N. B. Quashie, "Prevalence of congenital malaria in high-risk Ghanaian newborns: a cross-sectional study," Malaria Journal, vol. 12, no. 1, article 17, 2013.

[15] T. C. E. Mosha, D. Ntarukimana, and J. Matilda, "Prevalence of congenital malaria among newborn babies at Morogoro Regional Hospital, Morogoro, Tanzania," Tanzania Journal of Health research, vol. 12, no. 4, pp. 241-248, 2010.

[16] M. K. Mwaniki, A. W. Talbert, F. N. Mturi et al., "Congenital and neonatal malaria in a rural Kenyan district hospital: an eight-year analysis," Malaria Journal, vol. 9, article 313, 2010.

[17] P. R. Fischer, "Congenital malaria: an African survey," Clinical Pediatrics, vol. 36, no. 7, pp. 411-413, 1997.

[18] C. Falade, O. Mokuolu, H. Okafor et al., "Epidemiology of congenital malaria in Nigeria: a multi-centre study," Tropical Medicine and International Health, vol. 12, no. 11, pp. 12791287, 2007.

[19] E. M. Bah, I. S. Baldé, I. S. Diallo et al., "Congenital malaria and pregnancy monitoring parameters in health facilities in Guinea," Open Journal of Obstetrics and Gynecology, vol. 9, no. 1, pp. 73-82, 2019.

[20] P. J. Lehner and C. J. A. Andrews, "Congenital malaria in Papua New Guinea," Transaction of the Royals Society of Tropical Medicine and Hygiene, vol. 82, no. 6, pp. 822-826, 1988.

[21] P. O. Obiajunwa, J. A. Owa, and O. O. Adeodu, "Prevalence of congenital malaria in Ile-Ife, Nigeria," Journal of Tropical Pediatrics, vol. 51, no. 4, pp. 219-222, 2005.

[22] G. G. Sagbo, A. Noudamadjo, J. Agossou, J. D. Adedemy, A. A. Obossou, and L. D. Saturnin, "Epidemiological, clinical, biological, therapeutic features and outcome of congenital malaria at the Borgou Regional University Teaching Hospital (CHUD-B) in Benin in 2015," Open Journal of Pediatrics, vol. 7, no. 4, pp. 263-271, 2017.

[23] A. Noudamadjo, G. G. Sagbo, J. D. Adededmy, A. Joseph, A. A. Obossou, and L. D. Saturnin, "Frequency and sociodemographic factors associated with congenital malaria at the Borgou Regional University Teaching Hospital (CHUD-B) in Benin in 2015," Open Journal of Pediatrics, vol. 7, no. 4, pp. 215-221, 2017.

[24] J. Agossou, F. A. Mohamed, M. G. Kpanidja, A. Noudamadjo, J. D. Adédémy, and Z. R. Ahodègnon, "Congenital malaria in the Borgou/Alibori Regional University Teaching Hospital: myth or reality?," Open Journal of Pediatrics, vol. 9, no. 3, pp. 183-191, 2019.

[25] H. A. Vanga-Bosson, P. A. Coffie, S. Kanhon et al., "Coverage of intermittent prevention treatment with sulphadoxinepyrimethamine among pregnant women and congenital malaria in Côte d'Ivoire," Malaria Journal, vol. 10, no. 1, p. $105,2011$.

[26] O. N. Labama, N. J.-D. Bosenge, A. M.-A. Maindo et al., "Prevalence of gestational malaria in Kisangani, Democratic Republic of Congo," Open Journal of Obstetrics and Gynecology, vol. 8, no. 10, pp. 854-866, 2018.
[27] E. Ruh, J. P. Bateko, T. Imir, and A. Taylan-Ozkan, "Molecular identification of sulfadoxine-pyrimethamine resistance in malaria infected women who received intermittent preventive treatment in the Democratic Republic of Congo," Malaria Journal, vol. 17, article 17, 2018.

[28] O. N. Labama, N. J.-D. Bosenge, A. M.-A. Maindo, L. J. Losimba, and O. J.-P. Manga, "Intermittent preventive treatment in pregnancy with sulphadoxine-pyrimethamine does not have effect on maternal hemoglobin at delivery and birth weight in Kisangani, Democratic Republic of Congo," Journal of Biosciences and Medicines, vol. 7, no. 12, pp. 168-180, 2019. 